\title{
Randomized controlled trial of a web-based computer-tailored smoking cessation program as a supplement to nicotine patch therapy
}

\author{
Victor J. Strecher', Saul Shiffman ${ }^{2,3} \&$ Robert West ${ }^{4}$ \\ University of Michigan, Ann Arbor, USA', University of Pittsburgh, Pennsylvania, USA', Pinney Associates, LLC ${ }^{3}$ and University College London, London, UK
}

\author{
Correspondence to: \\ Victor J. Strecher \\ University of Michigan Comprehensive \\ Cancer Center \\ 300 N. Ingalls, Room 5D-04 (0471) \\ Ann Arbor \\ MI 48109-0471 \\ USA \\ Tel: 7347636099 \\ Fax: 7346477343 \\ E-mail: strecher@umich.edu \\ Submitted 22 June 2004; \\ initial review completed 16 August 2004; \\ final version accepted 22 November 2004
}

\begin{abstract}
Aim To assess the efficacy of World Wide Web-based tailored behavioral smoking cessation materials among nicotine patch users.

Design Two-group randomized controlled trial.

Setting World Wide Web in England and Republic of Ireland.

Participants A total of 3971 subjects who purchased a particular brand of nicotine patch and logged-on to use a free web-based behavioral support program.

Intervention Web-based tailored behavioral smoking cessation materials or web-based non-tailored materials.

Measurements Twenty-eight-day continuous abstinence rates were assessed by internet-based survey at 6 -week follow-up and 10-week continuous rates at 12-week follow-up.

Findings Using three approaches to the analyses of 6- and 12-week outcomes, participants in the tailored condition reported clinically and statistically significantly higher continuous abstinence rates than participants in the nontailored condition. In our primary analyses using as a denominator all subjects who logged-on to the treatment site at least once, continuous abstinence rates at 6 weeks were $29.0 \%$ in the tailored condition versus $23.9 \%$ in the nontailored condition $(\mathrm{OR}=1.30 ; \mathrm{P}=0.0006)$; at 12 weeks continuous abstinence rates were $22.8 \%$ versus $18.1 \%$, respectively ( $\mathrm{OR}=1.34 ; P=0.0006)$. Moreover, satisfaction with the program was significantly higher in the tailored than in the non-tailored condition.

Conclusions The results of this study demonstrate a benefit of the web-based tailored behavioral support materials used in conjunction with nicotine replacement therapy. A web-based program that collects relevant information from users and tailors the intervention to their specific needs had significant advantages over a web-based non-tailored cessation program.
\end{abstract}

KEYWORDS Internet, smoking cessation, nicotine replacement therapy, tailoring, web.

\section{INTRODUCTION}

Smoking is the greatest preventable cause of morbidity and mortality in the developed world (World Health Organization 2002)7. Because of the lag between smoking onset and disease occurrence, only cessation of smok- ing by current adult smokers is likely to yield reductions in mortality within the next 25 years (Doll et al. 1994). Ideally, smoking cessation programs should have the potential of reaching a large number of smokers, be easy to adopt, be effective, have good fidelity (preserve the program's original effectiveness during the implementation 
phase) and be easy to maintain over time (Glasgow et al. 1992). Most current antismoking efforts have been unable to offer all of these characteristics. Mass media approaches have wide reach but low effectiveness (Lichtenstein \& Glasgow 1992). On the other hand, intensive multi-session clinical interventions can achieve up to 28\% long-term cessation rates (Fiore et al. 2000), but their reach is limited (Schmid, Jeffrey \& Hellerstedt 1989; Fiore et al. 1990; Wagner et al. 1990).

Computer-based communication technologies hold the promise of combining the benefits of high-reach media-based interventions, individually orientated clinics and popular self-help programs. With the rapid development of such technology, tailoring a cessation program to the specific needs and interests of the smoker has become increasingly more sophisticated and less expensive (de Vries \& Brug 1999; Strecher 1999). Tailoring requires an assessment of a person's individual characteristics, as opposed to targeting, which is based on broader group identification (e.g. gender) (Kreuter, Strecher \& Glassman 1999; Strecher 1999). Tailoring is typically undertaken using computer programs that attempt to use rules for problems ordinarily requiring an expert. A commonly used program consists of: (a) an assessment of individual characteristics relevant to smoking cessation, (b) algorithms that use these data to generate interventions tailored to the specific needs of the user and (c) a feedback protocol that delivers these messages to the smoker in a clear, vivid form.

A generally positive body of evidence demonstrates the efficacy of print-based computer-tailored smoking cessation interventions in adults (Strecher 1999; Lancaster \& Stead 2002), and two trials have found higher cessation rates as a result of adding a tailored print-based program ('Committed Quitters ${ }^{\mathrm{TM}}$ ') to nicotine gum or patch pharmacotherapy (Shiffman et al. 2000, 2001). A key question is whether the effects of tailoring can be transferred to an even less costly, potentially high-reach channel, the World Wide Web. To our knowledge there are no published, randomized controlled trials of webbased smoking cessation programs (Lancaster \& Stead 2002). The present study represents the first study of this kind.

\section{SUBJECTS AND METHODS}

\section{Subjects}

Subjects for the study were recruited from smokers in the United Kingdom and Republic of Ireland who purchased NiQuitin CQ $21 \mathrm{mg}$ patch and connected to a website to enroll for free behavioral support materials. Eligible enrollees were required to meet the following criteria: (1) had a target quit date that was within 7 days from the enrollment date; (2) provided a valid e-mail address and had internet access for the duration of the study; (3) were attempting to quit smoking cigarettes (i.e. not smokeless tobacco); (4) had been smoking more than 10 cigarettes per day; (5) had purchased NiQuitin CQ $21 \mathrm{mg}$ (21 mg of nicotine, indicated for those who smoke at least 10 cigarettes per day); (6) agreed to be contacted for follow-up email and web-based questionnaires at 6 and 12 weeks; and (7) were 18 years of age or older. Eligible enrollees were invited to participate in the study; among those invited, $76.8 \%$ agreed to participate.

A total of 3971 subjects completed the baseline questionnaire and were randomized to one of two treatment conditions. Within 24 hours of completing the baseline questionnaire, all subjects were notified by e-mail that they had access to their behavioral support internet site. The majority of subjects were women $(56.5 \%)$; the average age was 36.9 years $(S D=10.2)$. Subjects smoked an average of 23.5 cigarettes per day $(\mathrm{SD}=8.2)$ and had smoked for an average of 20.1 years $(S D=10.3)$. The large majority of subjects $(88.4 \%)$ smoked their first cigarette of the day within the first 30 minutes after waking.

\section{Treatment conditions}

Enrollees were assigned randomly to one of two webbased treatment conditions: the active CQ PLAN or nontailored web-based smoking cessation materials. Participants were not informed of their treatment assignment. There were no significant differences in demographic or smoking history characteristics between subjects randomized to the CQ PLAN and control condition.

\section{Web-based Committed Quitters ${ }^{T M}$ Stop Smoking Plan (CQ PLAN)}

Information collected in the enrollment questionnaire was used to tailor the CQ PLAN materials (HealthMedia, Inc., Ann Arbor, MI, USA), including: demographics, smoking history, motives for quitting, expected difficulties quitting and situations that were expected to present challenges. In addition, subjects were allowed to identify a supportive person that would receive an e-mail message with tailored advice for supporting the subject. Program materials consisted of an initial web-based cessation guide, three sequential tailored newsletters delivered via the web and behavioral support messages delivered via email over a 10-week period. The content of the program was based on cognitive-behavioral methods of smoking cessation and relapse prevention, including stimulus control, self-efficacy enhancement, suggestions for coping and encouraging compliance with nicotine replacement therapy (Shiffman, Read \&AMP; Jarvik 1985; Fiore 
et al. 1990). Over $10^{30}$ different substantive combinations (i.e. combinations excluding numeric and namebased variables in the calculation) of the materials could be created. This represents a substantial increase over previous tailored cessation programs in tailoring depth or 'granularity' of the cessation advice provided. The format and volume of materials was equivalent for all CQ PLAN enrollees, but content and presentation was tailored based on enrollment information.

\section{Web-based control condition}

Web-based materials of the control condition had a graphic design and navigational structure very similar to the CQ PLAN. Cognitive-behavioral concepts similar to those addressed in the CQ PLAN were presented. Instruction on product use was also similar to instructions included in the CQ Plan. The primary differences were that subjects in the control condition did not receive: (a) tailored materials, i.e. enrollment data were not used to select the information or organize the information received by the user; (b) the three follow-up newsletters; and (c) the opportunity to identify a supportive person who subsequently received an e-mail message with tailored advice.

\section{Abstinence measures}

In the 6-week questionnaire, continuous abstinence was defined as self-report of no smoking at all for the previous 28 days. This is similar to generally accepted criteria used by numerous regulatory agencies around the world for assessing efficacy (US Food \& Drug Administration 1995). There was, however, no biochemical verification of self-reported abstinence; biochemical verification is considered impractical in large-scale population-based studies (Strecher et al. 1989; Patrick et al. 1994). At 12 weeks, continuous abstinence was defined as at least 10 weeks of abstinence.

\section{Data analysis}

Primary analyses focused on subjects who were randomized to one of the two treatment conditions and logged-on to the intervention website at least once (LO). Outcomes were also examined using an intent-to-treat (ITT) analysis (all subjects who were randomized to treatment conditions regardless of whether they logged-on to the intervention website) and an analysis including subjects who used the program and responded to the follow-up questionnaire (UPR). In the LO and ITT analyses, subjects who withdrew from the study or did not answer the abstinence questions were considered as treatment failures (that they did smoke). Subjects were included in the UPR population if they: (1) did not withdraw from the study; (2) answered the continuous abstinence question; (3) reported having read at least some of the materials; and (4) did not report having used other smoking cessation aids or programs.

Differences between treatment outcomes were assessed using logistic regression. A secondary multivariate model included age and baseline smoking rate as covariates. We also assessed whether enhanced compliance with nicotine replacement therapy (NRT) medication could have mediated the treatment effects. In other words, did the behaviorally based CQ PLAN treatment program per se have a direct effect on cessation and/or did the CQ PLAN have an indirect effect by increasing medication compliance, which in turn improved cessation outcomes? Shiffman et al. (2000) found that the printbased CQ PLAN intervention enhanced nicotine gum compliance but also demonstrated that cessation outcomes were not completely mediated by improved gum compliance.

Satisfaction, helpfulness and relevance with the two web-based treatments at the 6-week follow-up were also ascertained. Subjects were asked whether they would recommend the program to other smokers and, if they were smoking at the 6-week follow-up, whether they would use the program again in the future.

\section{Follow-up procedures}

Six weeks after their stated quit date, subjects were sent an e-mail to access a questionnaire which asked about their smoking abstinence, compliance with their program and patches and perceptions regarding their program. After a 2-week window, subjects were not permitted to respond but could still continue in the study. At 12 weeks after their stated quit date, subjects were sent a second questionnaire similar to the previous one, again with a 2 -week window.

Multiple e-mail reminders and compensation were provided to encourage completion of the follow-up questionnaires. Subjects completing either 6- or 12-week questionnaires received oral care products to the value of $£ 5$ (roughly \$9) (e.g. toothpaste, brushes). Subjects completing both 6 - and 12-week questionnaires received oral care products to the value of $£ 20$ (roughly \$35). Questions about abstinence were administered through an initial e-mail message instructing the subject to click one of two buttons - one button for 'did not smoke' or the other for 'did smoke' during the previous 28 days (6-week follow-up) or 10 weeks (12-week follow-up). When the subject clicked a button that response was automatically registered, and the subject brought to the website to complete the remainder of the questionnaire. Subjects who clicked 'did smoke' were then asked if they smoked during 
the previous 7 days (point prevalence). All respondents were asked questions regarding compliance and program satisfaction.

\section{Subject attrition}

Of the 3971 subjects enrolled in the study (constituting the ITT denominator), 1991 were randomized to the CQ PLAN and 1980 to the control condition. Of those randomized, 232 in the CQ PLAN and 238 in the control condition never logged-on to the website to receive their behavioral treatment. In the ITT analyses, subjects who never logged-on to the website were included in the denominator and considered to be smokers.

Of the 3501 who logged on to the website at least once, 31 subjects actively withdrew (15 in the CQ PLAN and 16 in the control condition) before the 6-week follow-up, leaving 3470 to receive the 6-week follow-up web-based survey. A total of 1850 (53.3\%) responded to the 6-week follow-up survey. Among the respondents, 37 subjects reported not having read the materials; and 552 reported using other smoking cessation aids or programs during the course of the study. These subjects were included in LO and ITT analyses but removed for the UPR analyses, which examined those who responded to follow-up, used the materials, and did not use other cessation treatments.

Between 6- and 12-week follow-up periods, 15 more subjects had withdrawn actively from the study (eight in the CQ PLAN and seven in the control condition), leaving 3455 subjects, of whom 1491 (43.2\%) responded to the 12-week survey. A total of 45 subjects reported not having read the materials and 471 reported using other smoking cessation aids or programs. As in the 6-week analysis procedure, these subjects were included in LO and ITT analyses but removed for the UPR analyses.

Response rates to the 6- and 12-week follow-ups did not differ between the two treatment conditions. Respondents did not differ from non-respondents by age or gen- der, but differed slightly in baseline smoking rate. On average, 6-week non-respondents smoked 0.8 cigarettes more than respondents $(F=8.1 ; P<0.01)$; 12-week nonrespondents smoked 1.0 cigarettes more than respondents $(F=13.7 ; P<0.001)$.

\section{RESULTS}

\section{Outcome analyses}

Table 1 presents 6 - and 12 -week outcomes by treatment condition. The primary 6-week LO analysis found a significantly higher 28-day continuous abstinence rate among subjects assigned to the CQ PLAN (29.0\%) than the control condition (23.9\%). Among the ITT subjects who enrolled in the study but did not necessarily log-on to the website to receive treatment, a significantly higher 6-week 28-day continuous abstinence rate was also found among CQ PLAN subjects $(25.6 \%)$ than control subjects $(21.0 \%)$. Significant differences between the CQ PLAN and control conditions remained at 12-week follow-up using both LO (22.8\% versus $18.1 \%)$ and ITT (20.1\% versus $15.9 \%$ ) analyses. UPR analyses also demonstrate consistently higher continuous abstinence rates in the CQ PLAN than the control condition at 6 weeks $(54.4 \%$ versus $46.8 \%)$ and 12 weeks $(55.4 \%$ versus 43.3\%). These outcomes did not change when baseline age and smoking rate were entered into the logistic model.

Subjects in the CQ PLAN group reported using nicotine patches for significantly longer over the course of the study (9.2 versus 8.5 weeks; $P<0.001$ ) Given that patch use typically stops when smokers relapse, this could be simply because those in the CQ PLAN group were more likely to remain abstinent. However, it is noteworthy that the effect of the CQ PLAN remained largely unaffected even after duration of patch use had been controlled for $\left(\mathrm{OR}=1.42\right.$ CI: $\left.1.15-1.74 ; \chi^{2}=10.7, P=0.001\right)$ showing

Table 1 Comparison of abstinence rates between CQ PLAN and control conditions.

\begin{tabular}{|c|c|c|c|c|c|c|c|}
\hline \multirow[b]{2}{*}{ Abstinence rates } & \multicolumn{2}{|c|}{ CQ PLAN } & \multicolumn{2}{|c|}{ Control } & \multirow[b]{2}{*}{ OR } & \multirow[b]{2}{*}{$95 \%$ CI of ORs } & \multirow[b]{2}{*}{ P-value } \\
\hline & $n$ & $\%$ & $n$ & $\%$ & & & \\
\hline \multicolumn{8}{|l|}{ Logged-on (LO) } \\
\hline 28-day abstinence at 6 weeks & 1759 & 29.0 & 1742 & 23.9 & 1.30 & $1.12-1.51$ & 0.0006 \\
\hline 10-week abstinence at 12 weeks & 1759 & 22.8 & 1742 & 18.1 & 1.34 & $1.13-1.58$ & 0.0006 \\
\hline \multicolumn{8}{|l|}{ All randomized (ITT) } \\
\hline 28-day abstinence at 6 weeks & 1991 & 25.6 & 1980 & 21.0 & 1.29 & $1.12-1.50$ & 0.0006 \\
\hline 10-week abstinence at 12 weeks & 1991 & 20.1 & 1980 & 15.9 & 1.33 & $1.13-1.57$ & 0.0005 \\
\hline \multicolumn{8}{|l|}{ Used program and responded (UPR) } \\
\hline 28-day abstinence at 6 weeks & 640 & 54.4 & 588 & 46.8 & 1.36 & $1.08-1.70$ & 0.008 \\
\hline 10-week abstinence at 12 weeks & 446 & 55.4 & 418 & 43.3 & 1.63 & $1.24-2.13$ & 0.0004 \\
\hline
\end{tabular}


that the CQ PLAN effect was not mediated by increased patch use.

\section{Program satisfaction}

On the 6-week questionnaire, respondents in the CQ PLAN condition were more likely than those in the control condition to report the materials as being helpful and relevant (Table 2), and more likely to state they would recommend the program to others. Among those who were smoking at the 6-week follow-up, CQ PLAN respondents were more likely to state that they would use the program in the future.

\section{DISCUSSION}

This study demonstrated the efficacy of the tailored webbased CQ PLAN behavioral smoking cessation program over a non-tailored web-based program in subjects using nicotine patches. Using three different analysis strategies, differing in whether subjects logged-on to the web site, used the program materials, did not use other cessation treatments or responded to follow-up, subjects randomized to the CQ PLAN condition reported significantly higher rates of cessation at both 6- and 12-week followup. Moreover, the effects on cessation from the tailored CQ PLAN were above and beyond any effects related to nicotine patch use.

The effect sizes of this intervention are comparable to those found in print-based tailored materials studies. In a meta-analysis of print-based tailored versus non-tailored self-help smoking cessation materials (Lancaster \& Stead 2002 ) found an average odds ratio of 1.36 (CI: 1.131.64) for computer-tailored materials compared with non-tailored or stage-matched materials (10 studies). As a point of comparison, Lichtenstein et al. (1996) found an average odds ratio of 1.34 (CI: 1.19-1.51) for telephone counseling compared with control conditions (13 studies).

The effects found in this study are also consistent with the results of two trials of print-based computer-tailored behavioral materials added to nicotine replacement therapy. In one study (Shiffman et al. 2000), provision of the tailored print Committed Quitters program with nicotine gum therapy significantly increased quit rates compared to the use of nicotine gum with prepackaged instructions and cessation advice alone ( 6 weeks: 36.2 versus $24.7 \%$; 12 weeks: 27.6 versus $17.7 \%$ ). In a similar study of the print Committed Quitters program with the nicotine patch (Shiffman et al. 2001), abstinence rates differed among those who reported using their assigned materials (over $80 \%$ of the sample -6 weeks: 38.2 versus $30.7 \%$; 12 weeks: 18.2 versus $11.1 \%$ ).

While the efficacy of these three approaches appears comparable, the costs differ substantially. The costs of commercial telecounseling products usually range between $\$ 150$ and $\$ 250$ per smoker; tailored print products usually vary between $\$ 5$ and $\$ 40$ per smoker and tailored web products, depending on size of the population, can be less than $\$ 1$ per smoker. Thus the web may offer a relatively high-reach, high-efficacy, low-cost approach to population-based smoking cessation.

Web-based programs may also have the potential for reaching large numbers of smokers (Bock et al. 2004). The number of adult web users in the world now exceeds half a billion (NUA Internet Survey 2003). Over 60\% of individuals in the United Kingdom (NUA Internet Survey 2003 ) and over $70 \%$ of individuals in the United States (UCLA Internet Report 2003) use the internet. The Pew Internet and American Life Project survey conducted in late 2002 (Fox \& Fallows 2003) found roughly 7 million individuals ( $6 \%$ of US internet users) searching the web for information on how to quit smoking. This reach appears to compare favorably with an estimated 500 000-700 $000(1.1-1.7 \%)$ of adult smokers calling a quitline over the course of a year (Ossip-Klein \& McIntosh 2003).

The effects of the CQ PLAN intervention in the study were incremental to the well-known effects of nicotine patch itself in enhancing abstinence (Silagy et al. 2004). One objective of the program was to enhance compliance with use of the nicotine patch, and smokers in the CQ PLAN were indeed found to use patches for longer. Whether this was because of compliance interventions in the CQ PLAN or just because they were abstinent longer is not known. In any case, the analysis showed that the CQ PLAN's effects on abstinence were not mediated by

Table 2 Satisfaction with the two treatments at 6-week follow-up (\% affirmative).

\begin{tabular}{lccc}
\hline & CQ PLAN & Control & $\chi^{2} ;$ P-value \\
\hline Internet materials helpful? & $85.9 \%$ & $68.9 \%$ & $\chi^{2}=108.6 ; P<0.0001$ \\
Personally relevant? & $88.9 \%$ & $69.8 \%$ & $\chi^{2}=160.2 ; P<0.0001$ \\
Recommend program to others? & $93.9 \%$ & $82.5 \%$ & $\chi^{2}=110.4 ; P<0.0001$ \\
Use program in the future?* & $91.4 \%$ & $79.2 \%$ & $\chi^{2}=26.0 ; P<0.0001$ \\
\hline
\end{tabular}

*Question only asked of subjects still smoking at follow-up. 
increased patch use, but derived from the advice and support conveyed by the material.

The current study should not be considered a direct test isolating the effects of tailoring on the efficacy of smoking cessation materials. The CQ PLAN had more waves of interaction with the user than the non-tailored control condition and it is possible that the additional interactions may have created the effect on smoking cessation. However, a direct test of the effect of increasing mailings of tailored materials failed to demonstrate effects on cessation (Velicer et al. 1999). The CQ PLAN-but not the control condition - also allowed the subject to identify a supportive person who would subsequently receive an email message with tailored advice for supporting the subject. While this addition may have accounted for improvements in cessation, a review of research studying the impact of buddy systems (May \& West 2000) found only two of 10 such studies to demonstrate a significant effect on cessation. None the less, a control condition that allows an adequate test of tailoring per se would require far greater structural similarities to the tailored condition.

This study could not verify claims of abstinence biochemically because of the geographical spread of the sample. However, false reporting is considered to be minimal where there is no personal contact with a therapist (Strecher et al. 1989; Velicer et al. 1992). The high rate of loss to follow-up is also a limitation. However, the loss to follow-up was similar to that typically found in largescale smoking cessation trials. In our ITT analysis all subjects lost to follow-up were considered to be smokers, possibly making the ITT success rates unduly low. There are many possible reasons for loss to follow-up (e.g. lost access to e-mail or internet; blockage of e-mail reminders due to so-called 'spam detectors'). Regardless, the observed difference between the treatment conditions could not be explained by dropout, as there was no difference in dropout rates and a significant treatment effect was observed.

Another limitation of the study is the relatively short follow-up period. Relapse curves from smoking cessation trials indicate that it is possible to extrapolate from 10 weeks of continuous abstinence to long-term success with relapse rates being the same in intervention and control conditions (Hughes et al. 2003). Thus, the odds ratio indicating the relative efficacy of the CQ PLAN and control interventions should be generalizable, although the absolute abstinence rates would be expected to diminish over time. Future web-based studies should examine longer-term abstinence rates.

To our knowledge, this is the first large-scale trial of an automated web-based smoking cessation intervention. Results of this study demonstrate a promising short-term outcome of tailored web-based programming in conjunction with nicotine replacement therapy.

\section{Acknowledgements}

This research was supported by GlaxoSmithKline. The authors would like to sincerely thank Joseph Matthias, Carolyn Dresler, Michael Durcan, Mitchell Kotler, Aviette Musin, Al Zielke and Ted Dacko for their assistance and support of this research.

\section{Declaration of interest}

Dr Strecher is a shareholder in HealthMedia, Inc., which developed the intervention tested in this study and has undertaken consultancy and research for, and travel funds from, manufacturers of smoking cessation products including the sponsor of this study. Dr Shiffman serves as a consultant to GSKCH on an exclusive basis regarding matters relating to smoking cessation and also has a financial interest in a venture to develop a new nicotine replacement product. Dr West has undertaken consultancy and research for, and travel funds and hospitality from, manufacturers of smoking cessation products including the sponsor of this study.

\section{References}

Bock, B. C., Graham, A. L., Sciamanna, C. N., Krishnamoorthy, J., Whiteley, J., Carmona-Barros, R., Niaura, R. S. \& Abrams, D. B. (2004) Smoking cessation treatment on the Internet. Content, Quality, and Usability Nicotine and Tobacco Research, 6, 207-219.

Doll, R., Peto, R., Wheatley, K., Gray, R. \& Sutherland, I. (1994) Mortality in relation to smoking: 40 years' observations on male British doctors. BMJ, 309, 901-911.

Fiore, M. C., Novotny, T., Pierce, J., Giovino, G., Hatziandreu, E. J., Newcomb, P. A., Surawicz, T. S. \& Davis, R. M. (1990) Methods used to quit smoking in the United States: do cessation programs help? JAMA, 263, 2760-2765.

Fiore, M. C., Thompson, S. A., Lawrence, D. L., Welsch, S., Andrews, K., Ziarnik, M., Korberly, B., Englund, E., Schensky, A. E. \& Baker, T. (2000) Helping Wisconsin women quit smoking: a successful collaboration. Wisconsin Medical Journal, 99, $68-72$.

Fox, S. \& Fallows, D. (July 16, 2003) Internet Health Resources: health searches and E-mail have become more commonplace, but there is room for improvement in searches and overall Internet access. Pew Internet and American Life Project. Available at: http://www.pewinternet.org.

Glasgow, R. E., Mullooly, J. P., Vogt, T. M., Stevens, V. J., Lichtenstein, E., Hollis, J. F., Lando, H. A., Severson, H. H., Pearson, K. A. \& Vogt, M. R. (1992) Biochemical validation of smoking status in public health settings: pros, cons, and data from four low intensity intervention trials. Addictive Behaviors, 18, 504527.

Hughes, J. R., Keely, J. P., Niaura, R. S., Ossip-Klein, D. J., Richmond, R. L. \& Swan, G. E. (2003) Measures of abstinence in clinical trials: issues and recommendations. Nicotine and Tobacco Research, 5, 13-25.

Kreuter, M. W., Strecher, V. J. \& Glassman, B. (1999) One size does not fit all: The case for tailoring print materials. Annals of Behavioral Medicine, 2, 276-283. 
Lancaster, T. \& Stead, L. F. (2002) Self-help interventions for smoking cessation. Cochrane Database Systematic Reviews, 3, CD001118.

Lichtenstein, E. \& Glasgow, R. E. (1992) Smoking cessation: what have we learned over the past decade? Journal of Consulting and Clinical Psychology, 60, 518-527.

Lichtenstein, E., Glasgow, R. E., Lando, H. A., Ossip-Klein, D. J. \& Boles, S. M. (1996) Telephone counseling for smoking cessation: rationales and meta-analytic review of evidence. Health Education Research, 11, 243-257.

May, S. \& West, R. (2000) Do social support interventions ('buddy systems') aid smoking cessation? A review. Tobacco Control, 9, 415-422.

NUA Internet Survey (2003) Global Net Population Increases. Accessed 25 February 2003. Available at: http:// www.nua.com/surveys.

Ossip-Klein, D. J. \& McIntosh, S. (2003) Quitlines in North America: evidence base and applications. American Journal of Medical Sciences, 326, 201-205.

Patrick, D. L., Cheadle, A., Thompson, D. C., Diehr, P., Koepsill, T. \& Kinne, S. (1994) The validity of self-reported smoking: a review and meta-analysis. American Journal of Public Health, 84, 1086-1093.

Schmid, T. L., Jeffery, R. W. \& Hellerstedt, W. L. (1989) Direct mail recruitment to home-based smoking and weight control programs: a comparison of strategies. Preventive Medicine, 18, 503-517.

Shiffman, S., Paty, J. A., Rohay, J. M., Di Marino, M. E. \& Gitchell, J. G. (2000) The efficacy of computer-tailored smoking cessation material as a supplement to nicotine polacrilex gum therapy. Archives of Internal Medicine, 160, 1675-1681.

Shiffman, S., Paty, J. A., Rohay, J. M., Di Marino, M. E. \& Gitchell, J. G. (2001) The efficacy of computer-tailored smoking cessation material as a supplement to nicotine patch therapy. Drug and Alcohol Dependence, 64, 35-46.

Shiffman, S., Read, L. \& Jarvik, M. E. (1985) Smoking relapse situations: a preliminary typology. International Journal of the Addictions, 20, 311-318.
Silagy, C., Lancaster, T., Stead, L., Mant, D. \& Fowler, G. (2004) Nicotine replacement therapy for smoking cessation. Cochrane Database Systematic Reviews, 3, CD000146.

Strecher, V. J. (1999) Computer-tailored smoking cessation materials. Patient Education and Counseling, 36, 107-117.

Strecher, V. J., Becker, M. H., Clark, N. M. \& Prasada-Rao, P. (1989) Using patients' descriptions of alcohol consumption, diet, medication compliance, and cigarette smoking: the validity of self-reports in research and practice. Journal of General Internal Medicine, 4, 160-166.

UCLA Internet Report (2003) Surveying the Digital Future. Los Angeles, California: UCLA Center for Communication Policy. Accessed February 2003. Available at: http:// www.ccp.ucla.edu.

US Food and Drug Administration (1995) Transcript of the Joint Meeting of the Nonprescription Drugs Advisory Committee and the Drug Abuse Advisory Committee of the Food and Drug Administration. Rockville, MD: US Food and Drug Administration.

Velicer, W. F., Prochaska, J. O., Fava, J. L., Laforge, R. G. \& Rossi, J. S. (1999) Interactive versus noninteractive interventions and dose-response relationships for stage-matched smoking cessation programs in a managed care setting. Health Psychology, 18, 21-28.

Velicer, W. F., Prochaska, J. O., Rossi, J. S. \& Snow, M. G. (1992) Assessing outcome in smoking cessation studies. Psychological Bulletin, 111, 23-41.

de Vries, H. \& Brug, J. (1999) Computer-tailored interventions motivating people to adopt health promoting behaviors: introduction to a new approach. Patient Education and Counseling, 36, 99-105.

Wagner, E. H., Schoenbach, V. J., Orleans, C. T., Grothaus, L. C., Saunders, K. W., Curry, S. \& Pearson, D. C. (1990) Participation in a smoking cessation program: a populationbased perspective. American Journal of Preventive Medicine, 6, 258-266.

World Health Organization (2002) World Health Report. Geneva: World Health Organization. 